\title{
Enlightenments from researches and predictions of 2014-2016 super El Niño event
}

El Niño is a remarkable climate phenomenon with a basinwide warming of sea surface temperatures (SST) in the easterncentral tropical Pacific. El Niño means The Little Boy, or Christ Child in Spanish, and on the contrary, a basinwide cooling of the tropical Pacific SST is called La Niña that means The Little Girl in Spanish. Always, a large-scale SST change in the tropical Pacific are accompanied with an east-west oscillation of sea level pressure in atmosphere, named the Southern Oscillation. Thus, El Niño and Southern Oscillation are regarded as the same phenomenon but different manifestations in ocean and atmosphere, and usually called ENSO for short. As a quasi-periodic oscillatory phenomenon with a time scale of 2-7 years, ENSO has been well known as the dominant mode of interannual climate variability.

The impact of ENSO is worldwide as induced by the large-scale SST warming (El Niño) or cooling (La Niña) in eastern-central tropical Pacific which can transport so much heat into atmosphere as to drive atmospheric circulation anomalies in tropics and extratropics and further influence weather and climate. This makes the ENSO phenomenon be always focused on by international community in the past decades. Great progress has been made in understanding and predicting ENSO, particularly in its typical spatio-temporal characteristics, physical mechanisms, climate impacts, and prediction methodology. Until now, ENSO has been widely recognized as the climate phenomenon with the highest level of cognition, theory, and prediction.

However, many studies have suggested that ENSO behaviors are becoming more and more complicated. The center of SST anomalies for the canonical ENSO lies in the eastern Pacific (EP, viz. the cold-tongue region), while it tends to shift westward after 1980. The non-canonical type of ENSO, which is usually called central Pacific ENSO (CP ENSO) or warm-pool ENSO, has occurred more frequently in the central tropical Pacific (the edge of the warm pool region). In recent years, a number of published articles in the world are focusing on the characteristics, physical mechanisms, climate impacts, and future changes of CP ENSO. Increasing studies of this CP type drive a new round of research on ENSO (Yeh et al., 2014).

The 2014-2016 El Niño event has been recognized as another super event since 1982/1983 and 1997/1998 super El Niño events. According to the new national standard of China, the peak intensity of this event has been identified as the strongest since 1950 (Ren et al., 2017). Signals of this event appeared in early 2014, and after two fluctuations it eventually developed into a super event at the end of 2015. This super event caused severe flooding and drought disasters in many countries including China, which induced reduction of grain production and loss of lives and property.

Indeed, the latest event was quite different from the previous two super events, and both its distinctness and complexity have profoundly influenced some traditional understandings of us regarding typical characteristics and mechanisms of ENSO. For example, this super event had a clear eastward propagation of SST anomalies in the equatorial Pacific during its developing stage, but although it is regarded as EP type, its SST anomaly center was actually located in the middle of traditional positions of the EP and CP ENSO. Moreover, during its long lifetime, this event has experienced some complicated development processes, which brings a large challenge not only for the monitoring and forecasting at different stages of this unique event, but also for the traditional cognition and theoretical mechanisms of ENSO. Therefore, the distinctness of this super event has push forward the current round of ENSO research.

Four articles are selected in this special issue, which examine different aspects of the 2014-2016 El Niño event, including its evolution processes, dynamic mechanism, subsurface influence, and spring predictability barrier, respectively. These results

Citation: $\quad$ Mu M, Ren H L. 2017. Enlightenments from researches and predictions of 2014-2016 super El Niño event. Science China Earth Sciences, 60: 1569-1571, doi: $10.1007 / \mathrm{s} 11430-017-9094-5$ 
are still not enough for us to fully understand the distinct characteristics and complexity of this super event, it has no doubt that more studies are definitely needed for further examining its evolution in observation, diagnosing its mechanism, as well as conducting experiments of simulation and prediction in models. Anyway, ENSO is the most concerned climate phenomenon in the global high-impact environment events with air-sea interaction process. Thus, the editor expects that these articles in this issue can provide a useful reference for improving ENSO research and prediction, and further bring readers enlightenment and thinking.

\section{The distinct characteristics of the 2014-2016 super EI Niño event}

The diagnostic analysis, based on several international ocean reanalysis datasets in which variables include SST, sea surface wind, longwave radiation, subsurface sea temperature etc., showed that the 2014-2016 event began in 2014 autumn and SST anomalies in Niño3.4 region peaked in November 2015. The central Pacific warming reached a record high and the corresponding enhanced convection shifted westward apparently. During the development stage of this event, several downwelling Kelvin waves emerged and contributed to the fast warming in the equatorial central-eastern Pacific, while the rapid damping of this event might be closely related to the upwelling Kelvin waves appeared in November 2015 (Xue and Kumar, 2017).

The 2014-2016 El Niño event experienced two distinct developing stages, the weak El Niño stage and the strong developing stage. The former was in the autumn and winter of 2014, and the latter in the second half of 2015. The comparison of dynamical mechanisms between these two stages showed that the amount of westerly wind bursts (WWBs) in the western tropical Pacific might be the main reason of the significant differences in the warming intensity during the two stages. In addition, the diagnosis based on an ocean reanalysis dataset showed that the eastward transport of surface oceanic heat in the equatorial central Pacific and the vertical heat transport in the equatorial eastern Pacific also directly contributed to the formation of this super event (Lian et al., 2017).

In the development stage of 2014-2016 El Niño episode, the positive subsurface ocean temperature anomalies in the equatorial central-eastern Pacific were remarkably horseshoe-shaped distribution. Zhang and Gao (2017) investigated this significant feature by utilizing observation data combined with an intermediate ocean model. They mainly focused on the two processes which influenced the evolution of SST anomalies, viz., the remote effects connected with the propagation and reflection of equatorial waves in the boundary layer of ocean in 2015, and the local effects of the positive anomalous heating in the subsurface layer. Their results showed that the continuously positive anomalous heating in the subsurface layer had an important influence on maintenance of the positive SST anomalies in the equatorial Pacific, which help to deepen our understanding for this super event.

The characteristics of error growth were further investigated through using an intermediate prediction model. The significant spring predictability barrier (SPB) was found to occur when using this model to predict the 2014-2016 El Niño event, which was presumably induced by model error (Qi et al., 2017). The model error could be described by the SST tendency error which had a dipole structure over the equatorial Pacific with negative anomalies in the west and positive in the east. The structure of this tendency error was similar to that of the most sensitive nonlinear forcing singular vector-like tendency error. This kind of similar tendency error could lead to the SPB and most of prediction error in predictions of the previous two super El Niño events. As a result, this model may be corrected by the nonlinear forcing singular vector-like tendency error to further improve its prediction skill on super El Niño events.

\section{Enlightenments and thinking}

Undoubtedly, there are new issues and challenges to ENSO research and prediction from the complexity and diversity of ENSO events, particularly from the distinctness of this 2014-2016 El Niño event. It is unlikely to thoroughly address the problems and challenges that we are facing by only pinning our hopes to the papers in this special issue. However, the enlightenments and further thinking from this super event and related researches are worthy of note.

(1) Research and implementation of ocean target observation. Observation is the base of recognizing special phenomenon and studying physical mechanism. Numerical model has become a fundamental tool to perform forecast and prediction, and a large amount of available field observations contribute to the initial and boundary conditions of model. In the past three decades, several programs and campaigns of global oceanic observation were carried out. Although increasingly rich oceanic observations have been collected, it is still far from enough to serve for ENSO research and prediction (e.g., Xue and Kumar, 2017). To economically and effectively improve prediction skills of high-impact ocean-atmospheric environmental events, a large number of theoretical and numerical studies showed that it is feasible to perform deliberate oceanic target observations in terms of research or forecasting objectives (Mu et al., 2015). It is thus reasonable to expand our efforts to carrying out all-around studies and 
practices of ocean-atmosphere target observations by learning from typhoon target observations and implementations. For the super El Niño events like the 2014-2016 case, besides improving and optimizing conventional observation networks (e.g., the TAO/TRITON network), we should perform some extra intensive field target observations to generate better initial and boundary conditions for ENSO prediction, and further improve its prediction skill, just as the operational typhoon target observation. Meanwhile, to make full use of the conventional and target observation data, we should devote to the data assimilation methods and theoretical researches based on ocean-atmosphere coupled model.

(2) Fully coupled ocean-atmosphere models are needed for future theoretical research and prediction of ENSO. Significant distinctness and complexity has been observed in this event in terms of its fundamental characteristics and mechanisms of oceanatmosphere coupling system, compared with the previous two super El Niño events (Lian et al., 2017; Qi et al., 2017; Ren et al., 2017). It is not enough to give a comprehensive interpretation for this super event by exclusively using conventional linear ENSO dynamical theories, implying that the nonlinear processes of ocean-atmosphere system may be responsible for these distinctness and complexities. In addition, different from the previous two super events, this event displayed some unique characteristics in ocean subsurface and physical processes evolutions. Meanwhile, lots of WWB events that contributed to the excitation of ENSO signals have emerged intensively, which implies the importance of atmospheric signals. All these facts clearly tell us that improving ocean-atmosphere coupled models is the only way to simulate the complex nonlinear physical processes satisfactorily, for which the findings in previous studies concluded from the simplified or intermediate air-sea coupled climate models are undoubtedly valuable references. In the long term, it is an inevitable choice to develop fully coupled oceanatmosphere models for ENSO theoretical research and prediction.

\section{References}

Yeh S, Kug J, An S. 2014. Recent progress on two types of El Niño: Observations, dynamics, and future changes. Asia-Pac J Atmos Sci, 50: 69-81 Ren H L, Sun C H, Ren F M, Yuan Y, Lu B, Tian B, Zuo J Q, Liu Y, Cao L, Han R Q, Jia X L, Liu C Z. 2017. Identification method for El Niño/La Niña events. The People's Republic China's National Standard GB/T 33666-2017, May 2017. Beijing: Standards Press of China. 1-6

Xue Y, Kumar A. 2017. Evolution of the 2015/16 El Niño and historical perspective since 1979. Sci China Earth Sci, 60: 1572-1588, doi: 10.1007/s11430016-0106-9

Lian T, Chen D K, Tang Y M. 2017. Genesis of the 2014-2016 El Niño events. Sci China Earth Sci, 60: 1589-1600, doi: 10.1007/s11430-016-8315-5

Zhang R H, Gao C. 2017. Processes involved in the second-year warming of the 2015 El Niño event as derived from an intermediate ocean model. Sci China Earth Sci, 60: 1601-1613, doi: 10.1007/s11430-016-0201-9

Qi Q Q, Duan W S, Zheng F, Tang Y M. 2017. On the "spring predictability barrier" for strong El Niño events as derived from an intermediate coupled model ensemble prediction system. Sci China Earth Sci, 60: 1614-1631, doi: 10.1007/s11430-017-9087-2

Mu M, Duan W S, Chen D K, Yu W D. 2015. Target observations for improving initialization of high-impact ocean-atmospheric environmental events forecasting. Nat Sci Rev, 2: 226-236

Ren H L, Wang R, Zhai P M, Ding Y H, Lu B. 2017. Upper-ocean dynamical features and prediction of the super El Niño in 2015/16: A comparison with 1982/83 and 1997/98. J Meteor Res, 31: 278-294 